\title{
A Fire Monitoring Cloud Platform Based on Internet of Things
}

\author{
Fei-Xiang Xu, Jian-Jun Yi, Long De, Xiao-Ming Zhu \\ Department of Mechanical Engineering, East China University of Science and Technology, ECUST, Shanghai, China; \\ E-mail: xufeixiangsdut@163.com, jjyi@ecust.edu.cn, delongecust@foxmail.com, zxmin4236@163.com
}

\begin{abstract}
Based on the research of existing fire monitoring system, a fire monitoring cloud platform for monitoring building fire protection equipment status using Internet of Things (IoT) technology is proposed in this paper. The cloud platform consists of three layers: sensing communication layer, cloud computing center and application service layer. The first layer is responsible for collecting and transmitting the fire data. The second layer implements the physical resource virtualization operation and management of resource scheduling. The third layer provides users with the fire monitoring software as a service (SaaS) in the form of PC application and mobile application (APP). A demonstration of the cloud platform for building fire protection equipment status monitoring is validated in this paper. The results proved that this cloud platform not only can accurately monitor the fire protection equipment status of the building, but also presents important advantages such as generality, feasibility and expansibility.
\end{abstract}

\section{Keywords-fire monitoring; IoT; cloud computing center; SaaS}

\section{INTRODUCTION}

With the continuous development of society, there are more and more fire hazards brought by increasingly complex building structure [1]. Currently, fire monitoring systems are independent of each other, and there is a lack of mutual linkage and information sharing between the systems. When there is a fire, the fire monitoring staffs also have operational errors such as underreporting and delayed reporting in the process of reporting the alarming information to the fire department [2]. Consequently, it is imminent to build remote fire monitoring systems.

The IoT is the extension of internet applications [3], and it is the internet for connecting different things, realizing informatization management, remote control and intelligent monitoring management. Therefore, it is necessary to integrate IoT technology with fire monitoring system. However, fire monitoring system based on IoT must have the ability of mass data storage and computing. In this paper, we describe how IoT and cloud computing work together can address the big data issues [4]. Meanwhile, this cloud platform is provided for users in the form of SaaS to save the cost of the data center construction and software development for users [5].

This paper aims to realize a remote fire monitoring system for monitoring fire protection equipment status throughout the day. Integrating IoT with cloud computing, a fire monitoring cloud platform is proposed in this paper.

\section{OVERALL ARCHITECTURE}

Fig. 1 shows the overall architecture of the proposed fire monitoring cloud platform. The cloud platform consists of three layers: sensing communication layer, cloud computing center and application service layer.

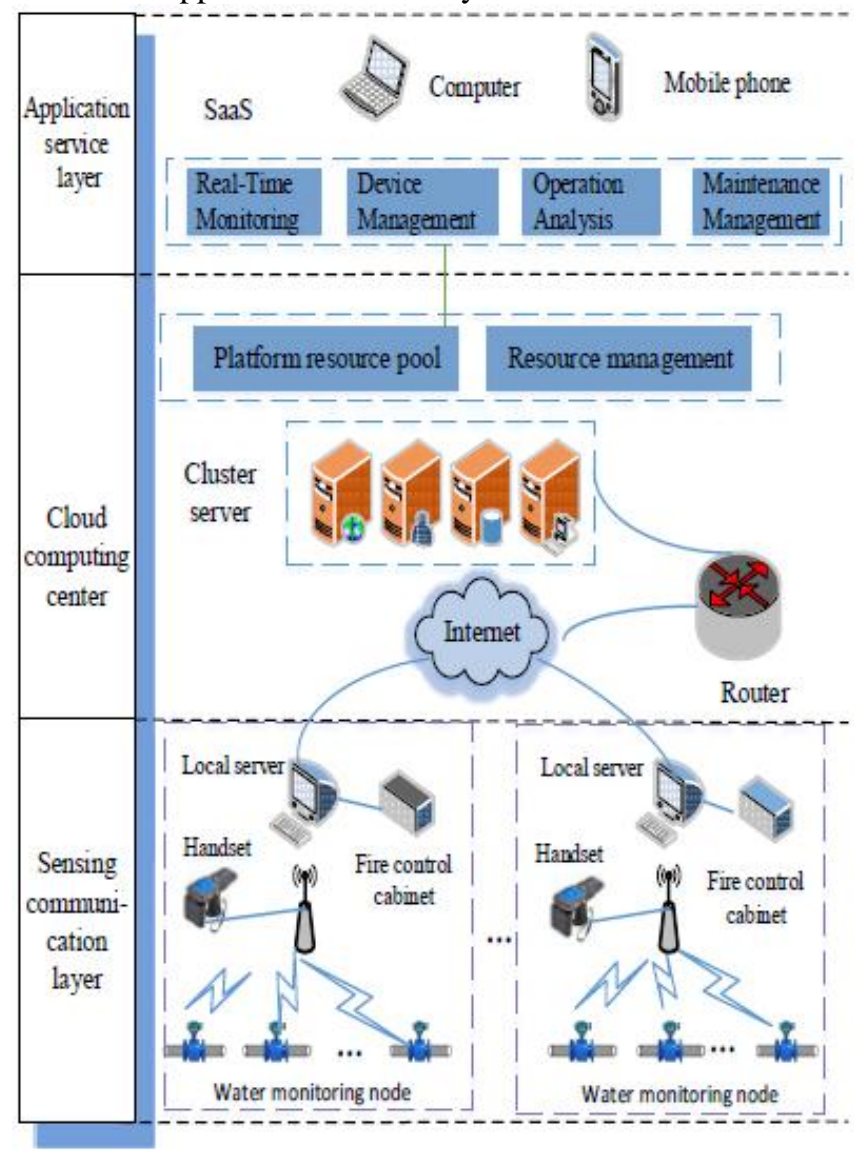

Figure 1. The overall architecture of the proposed cloud platform.

The data of sensing communication layer are derived from the IoT nodes for monitoring water pressure, RFID reading and writing equipment and fire control cabinet. After collecting the data of fire sprinkler system and fire deluge system, the water pressure nodes transfer the above data to the local server through the WIFI module, and then data will be sent to cloud computing center. RFID readwrite device is used in the fire protection facilities maintenance, and then maintenance information will be transferred to cloud computing center through the network. Fire control cabinet integrates the data of smoke detector, 
heat detector and alarm device. Then integrated data is transferred to the local server and then sent to cloud computing center.

The cloud computing center consists of the platform resource pool and resource management .Platform resource pool is responsible for the virtualization of physical resources. The resource management which includes resource allocation and task scheduling is mainly to realize the management of the virtual resources in the platform. The cloud computing center sets up infrastructure as a service providing the foundation platform for application service layer.

SaaS is provided for users who do not need to build the data center. The fire protection equipment status can be monitored remotely by users in the form of PC application and mobile APP. SaaS has four major functional modules, including real-time monitoring, device management, operation analysis and maintenance management.

\section{IMPLEMENTATION OF THE FIRE MONITORING CLOUD PLATFORM}

As described above, the fire monitoring cloud platform includes three layers. This paper mainly introduces the second layer and the third layer.

\section{A. Design of Cloud Computing}

Cloud computing center is the core of the whole fire monitoring cloud platform, which is responsible for the virtualization of physical resources and the scheduling management for virtual resources. As shown in Fig. 2, the cloud computing center provides the fundamental platform for application service layer, containing the platform resource pool and resource management. The platform resource pool converts server resources and storage resources of the physical layer to virtual server and virtual storage using virtual software [6].The resource management which includes resource allocation and task scheduling is responsible for realizing the management of virtualization resources in the platform. In this paper, resource management contains server management, virtual machine management, load balancing management, heterogeneous database systems management, application management and management of application deployment.

By taking steps of double machine hot standby scheme for domain control server with a single point of failure characteristics, server management is responsible for realizing server disaster preparedness, to avoid the fire alarming not being timely monitored because of the server's fails. Virtual machine management is mainly to add or delete a virtual machine, start up or shut down a virtual machine, etc. Heterogeneous database system management is mainly to design a heterogeneous database system by deploying some database systems in the different virtual machines, to achieve the storage of massive fire data. Application management is responsible for managing the data processing (receiving, parsing, and storage) application, cloud platform portal WEB application and fire monitoring WEB application. Application deployment management is to realize the above application's deployment. To relieve the
WEB server's load pressure, load balancing management is mainly to take the method of load balancing to bear WEB applications by creating multiple virtual machine [7].Hence, the huge loads of WEB application server brought by the large amount of fire data can be avoided.

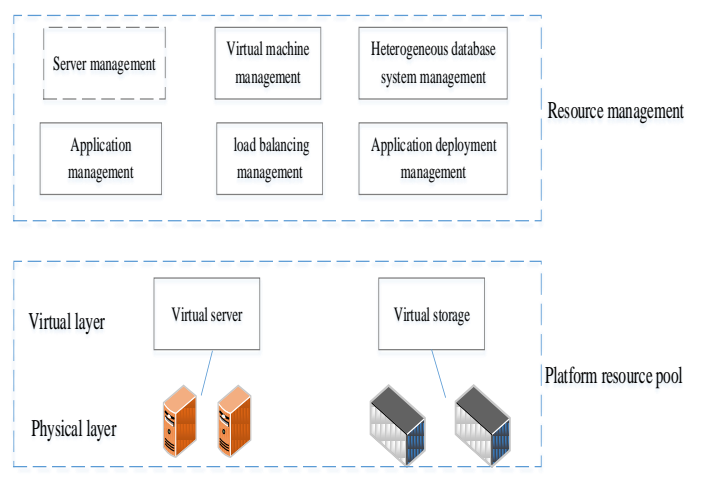

Figure 2. Design of cloud computing center.

\section{B. Design and Development of Application Service Layer}

The running flow chart of the application service layer is shown in Fig. 3.Firstly, users enter the portal site of cloud platform, and then they register the account and login. After that, they pay fees according to their using time, requirements, and their user types. Secondly, after the order received from the portal site, the cloud platform will sent staff to achieve its sensing communication layer, and then it will provide users with user permissions, username and password of fire monitoring system. Finally, users can monitor the fire protection equipment status in the fire monitoring system. When a user account balance is insufficient in cloud platform, he will be reminded. If the user doesn't charge, the cloud services for him will be stopped by cloud platform. Otherwise, he will continue to have the cloud services. As shown in Fig. 3, there are three kinds of user permissions including monitoring center, building units and the public security fire department in the cloud platform. Moreover, users with different permissions have different services. Next, the functional modules owned by monitoring center users will be discussed.

1) User management:

It consists of add or delete users, edit user information, and set user permissions.

2) Map show:

It identifies the location and real-time monitoring status of all monitored buildings. When clicking on the logo of the map, users can enter the real-time monitoring interface of corresponding building. :

\section{3) Real-time monitoring:}

It shows the real-time monitoring data to users in graphic interface with built three-dimensional models, and states of monitoring node are marked by tags with different color. When monitoring node status is normal, tags will be marked with blue; when monitoring node status is abnormal, tags will be marked with red. 


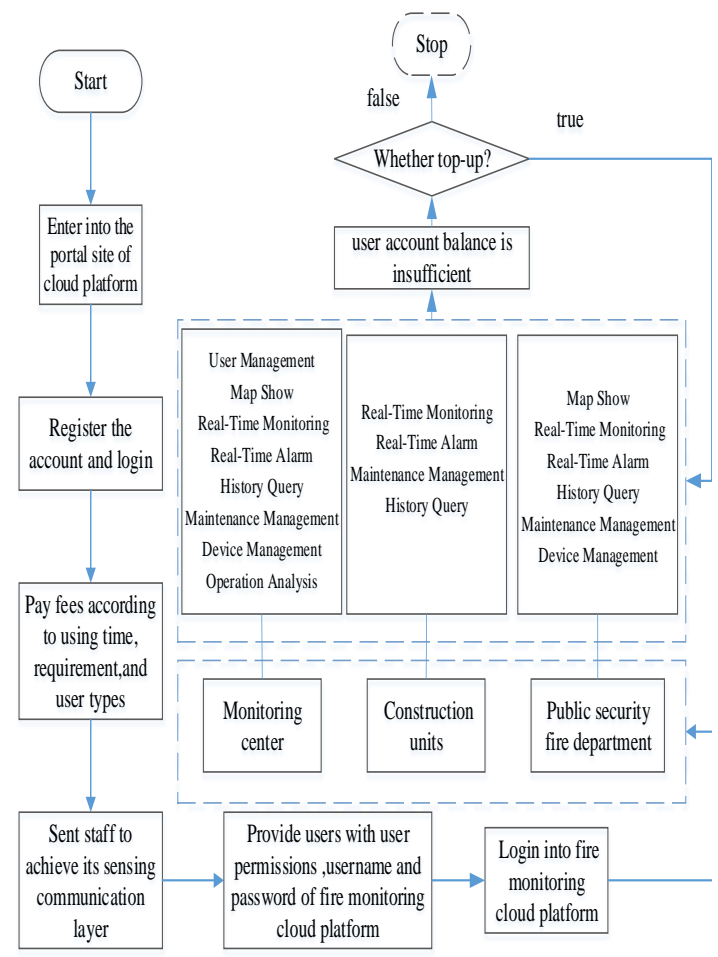

Figure 3. The running flow chart of application service layer

\section{4) Real-time alarm:}

It shows the latest alarm information of all the monitored nodes in the form of tables. When there is a fire alarm, the pop-up message, push notifications, and warning tone will be displayed to alert the users.

5) History query:

Submitting some information related to fire data, users can view historical data of the monitored nodes within a certain period of time.

6) Device management:

It is responsible for managing fire protection facilities, and it can display daily inspection information, daily maintenance information, and lost information of fire protection facilities.

\section{7) Maintenance management:}

It displays records of maintenance information and next maintenance's time of fire maintenance department.

8) Operation analysis:

The idea of big data is applied to analysis fire monitoring for all buildings. It displays the numbers of all constructions' alarm, all monitored node data trend, and the numbers of all building maintenance.

The application service layer provides users with above functional modules in the form of PC application and mobile APP. In order to monitor fire situation in the anytime and anywhere, PC application is developed based ASP.NET MVC website development technology using B/S (browser/server) architecture in the development environment of Windows server 2012R2 system. This platform builds the android APP and the iOS APP based on hybrid APP development technology with excellent crossplatform characteristics, to support popular mobile phone operation system.

\section{VALIDATION OF THE FIRE MONITORING CLOUD PLATFORM}

The fire monitoring cloud platform can work properly only when all layers (sensing communication layer, cloud computing center and application service layer) work well. Hence, in this paper, the monitoring of the fire protection equipment condition is taken as an example, to verify whether this platform is working properly. The fire data collected by fire nodes will be sent to the local server, and then sent to cloud monitoring center. Cloud computing center receives, parses and stores massive fire data in the heterogeneous database systems. The application service layer provides users with the fire monitoring software as a service (SaaS) in the form of PC application and mobile APP.

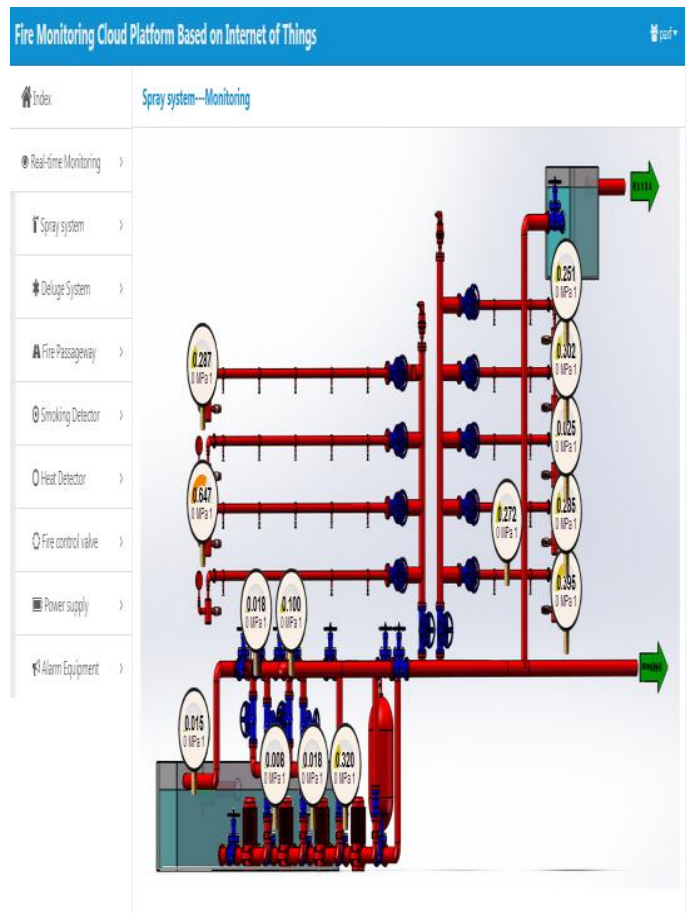

Figure 4. Real-time monitoring interface of PC application

The real-time monitoring interface of $\mathrm{PC}$ application with the monitoring center users is shown in Fig. 4. The statistics of alarming number interface of PC application with the monitoring center users is shown in Fig. 5.Some operation interfaces of IOS APP with the building unit users is shown in Fig. 6. In conclusion, with all layers working properly, the fire monitoring cloud platform based on IoT can accurately monitor the building fire protection equipment status, and provide different cloud services for users with different requirements. 


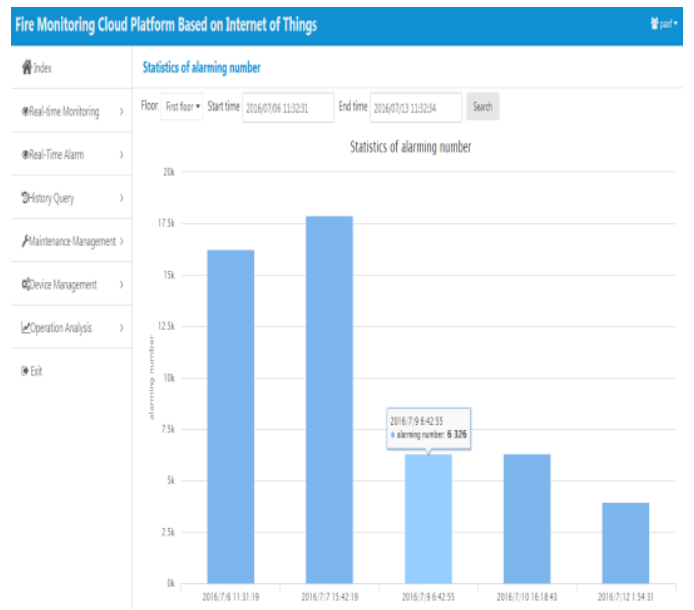

Figure 5. Statistics of alarming number interface of PC application

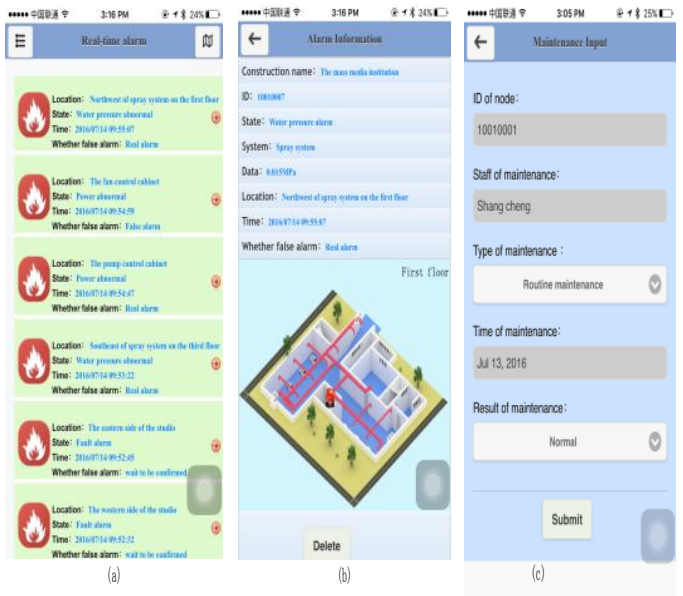

Figure 6. Interfaces of iOS APP: (a) real-time alarm (b) alarm information (c) maintenance input

\section{CONCLUSION}

Integrating IoT with cloud computing, the design and development of fire monitoring cloud platform are discussed in this paper. The building fire protection equipment status can be monitored from remote side. Further, the cloud platform saves the cost of the data center construction and software development for users who use cloud platform in the form of SaaS. Above all, because of the simple operation, flexible application and high stability, the fire monitoring cloud platform proposed in this paper, not only can be applied in the fire monitoring system of all buildings, but also can be widely used in the monitoring of some fields, such as the environment of farming production, the water quality and the equipment temperatures in substations and so on.

\section{ACKNOWLEDGMENT}

This paper was supported by the Research Foundation of Science and Technology Commission of Shanghai under Grant No. 10DZ1500200, the Natural Science Fund of China (NSFC) under Grant Nos. 50975088, 51275173, 51575186 and 51210105018, the Fundamental Research Funds for the Central Universities under Grant No. WH0913009, Shanghai Pujiang Program under Grant No. PJ201000353, and Shanghai Software and IC industry Development Special Fund under Grant No. 120493.

\section{REFERENCES}

[1] Rui-xiang Chen, Xiao-qiang Zhang, Chao-yang Peng, Hong-yong Zhang, "A Study on Design and Implementation of Remote Fire Monitoring System for Buildings," Procedia Engineering, vol. 52, Mar. 2013, pp. 56-59, doi:10.1016/j.proeng.2013.02.105.

[2] Zhang Hui, Chen Gudian, "City Fire Remote Monitoring System Based on Internet of Things," Informatization Research, vol. 36, Oct. 2010,pp .55-58, doi: 10.3969/j.issn. 1674-4888.2010.10.017.

[3] Ke Yin, JunCheng Jiang, "an Application of Internet of Things in the Field of Urban Building Fire Safety." International Journal of Safety and Security Engineering,vol.4,2010,pp.135-142,doi:10.2495/SAFEV4-N2-135-142.

[4] B. B Prahlada Rao, Paval Saluia, "Cloud computing for Internet of Things \& sensing based applications," Sensing Technology (ICST), 2012 Sixth International Conference on , IEEE Press Dec,2012,pp. 374 - 380, doi: 10.1109/ICSensT.2012.6461705.

[5] YU Pan, ZOU Chengjun, "Design on the Cloud Service Platform of Intelligent Greenhouse in Chengdu Based on Internet of Things," Agriculture Network Information, 2014, pp. 49-53, doi: 10.3969/j.issn. 1672-6251.2014.07.014.

[6] Fu yong Kang, "Environmental Private Cloud Platform Design and its SaaS Model Zongliang Jianpai Business System Implementation," 2013, University of Electronic Science and Technology of China.

[7] Li-Chuan Chen, Hyeong-Ah Choi, "Approximation Algorithms for Data Distribution with Load Balancing of Web Servers,"Cluster Computing, 2001. Proceedings. 2001 IEEE International Conference on,IEEE Press Oct.2001, doi:10.1109/CLUSTR.2001.959988. 